\title{
Geographical variations of the associations between health interventions and all-cause under-five mortality in Uganda
}

Betty B. Nambuusi ${ }^{1,2,3}$, Julius Ssempiira ${ }^{1,2,3}$, Fredrick E. Makumbi ${ }^{3}$, Jürg Utzinger ${ }^{1,2}$, Simon Kasasa ${ }^{3}$ and Penelope Vounatsou ${ }^{1,2^{*}}$ (i)

\begin{abstract}
Background: To reduce the under-five mortality (U5M), fine-gained spatial assessment of the effects of health interventions is critical because national averages can obscure important sub-national disparities. In turn, subnational estimates can guide control programmes for spatial targeting. The purpose of our study is to quantify associations of interventions with U5M rate at national and sub-national scales in Uganda and to identify interventions associated with the largest reductions in U5M rate at the sub-national scale.
\end{abstract}

Methods: Spatially explicit data on U5M, interventions and sociodemographic indicators were obtained from the 2011 Uganda Demographic and Health Survey (DHS). Climatic data were extracted from remote sensing sources. Bayesian geostatistical Weibull proportional hazards models with spatially varying effects at sub-national scales were utilized to quantify associations between all-cause U5M and interventions at national and regional levels. Bayesian variable selection was employed to select the most important determinants of U5M.

Results: At the national level, interventions associated with the highest reduction in U5M were artemisinin-based combination therapy (hazard rate ratio $(\mathrm{HRR})=0.60 ; 95 \%$ Bayesian credible interval $(\mathrm{BCl})$ : $0.11,0.79$ ), initiation of breastfeeding within $1 \mathrm{~h}$ of birth ( $\mathrm{HR}=0.70 ; 95 \% \mathrm{BCl}: 0.51,0.86)$, intermittent preventive treatment (IPTp) (HRR= 0.74; 95\% BCl: 0.67, 0.97) and access to insecticide-treated nets (ITN) (HRR $=0.75 ; 95 \%$ BCl: 0.63, 0.84). In Central 2, Mid-Western and South-West, largest reduction in U5M was associated with access to ITNs. In Mid-North and WestNile, improved source of drinking water explained most of the U5M reduction. In North-East, improved sanitation facilities were associated with the highest decline in U5M. In Kampala and Mid-Eastern, IPTp had the largest associated with U5M. In Central1 and East-Central, oral rehydration solution and postnatal care were associated with highest decreases in U5M respectively.

Conclusion: Sub-national estimates of the associations between U5M and interventions can guide control programmes for spatial targeting and accelerate progress towards mortality-related Sustainable Development Goals.

Keywords: Bayesian proportional hazards geostatistical models, Demographic and health survey, Geographical variations, Interventions, Sub-national scale, Uganda, Under-five mortality

\footnotetext{
* Correspondence: penelope.vounatsou@swisstph.ch

${ }^{1}$ Swiss Tropical and Public Health Institute, P.O. Box, CH-4002 Basel,

Switzerland

${ }^{2}$ University of Basel, P.O. Box, $\mathrm{CH}-4003$ Basel, Switzerland

Full list of author information is available at the end of the article
}

(c) The Author(s). 2019 Open Access This article is distributed under the terms of the Creative Commons Attribution 4.0 International License (http://creativecommons.org/licenses/by/4.0/), which permits unrestricted use, distribution, and reproduction in any medium, provided you give appropriate credit to the original author(s) and the source, provide a link to the Creative Commons license, and indicate if changes were made. The Creative Commons Public Domain Dedication waiver (http://creativecommons.org/publicdomain/zero/1.0/) applies to the data made available in this article, unless otherwise stated. 


\section{Background}

Under-five mortality (U5M) is an important indicator of the social and economic development of a specific country or a smaller administrative unit [1]. The government of Uganda has made progress in reducing the U5M; yet, it is still unacceptably high. According to the data obtained from the Demographic and Health Surveys (DHS), Uganda's U5M declined from 137 deaths per 1000 live births in 2006 to 90 in 2011 [2, 3]. Over the same 5-year period, the coverage of health interventions improved country-wide. For example, the percentage of children receiving vitamin A supplements in the past 6 months increased from 36 to $57 \%$. The percentage of children with fever 2 weeks prior to a survey who were given artemisinin-based combination therapy (ACT) increased from a mere 3\% in 2006 to $69 \%$ in 2011 . Coverage of other health interventions improved as well $[2,3]$.

Despite this progress at the national level, there are remarkable sub-national variations in $\mathrm{U} 5 \mathrm{M}$ and health intervention coverage. The lowest U5M rate (56 deaths per 1000 live births) and the highest (152 deaths per 1000 live births) were observed in Kampala and the North-East regions, respectively. The coverage of vitamin A supplements was $30 \%$ in Central 1, compared to $74 \%$ in the North-East. Skilled delivery varied from 31\% in the North-East to 93\% in Kampala. Such disparities may be associated with the observed discrepancies in regional mortality rates and thus need to be investigated at a regional scale.

In Uganda, there is a paucity of studies estimating the relation between $\mathrm{U} 5 \mathrm{M}$ and health interventions at a local scale. In addition, most studies that assessed the relationship between U5M and health interventions were not carried out at the national scale. For example, Bacillus Calmette Guerin (BCG) vaccination was associated with a lower rate of death among children aged between one and 5 years in a community-based prospective cohort study in the eastern part of Uganda [4]. A volunteer community health worker child promotion model was related to child mortality declines in rural South-West Uganda [5].

Household surveys provide a suitable source of national data to monitor progress of U5M and implemented interventions. A national DHS was conducted in Uganda in 2011, which provided the most reliable mortality and interventions data with national coverage. To date, a few national studies in Uganda used DHS data to quantify the relationship between health interventions and child mortality. However, these studies assessed only one category of child health interventions; namely, vaccinations [6]. Furthermore, previous research in other settings has used DHS data to evaluate effects of health interventions on U5M at the global [7] and subcontinental [8] scales. However, former studies did not take into account geographical variations in the coverage of health interventions that may influence U5M patterns [9]. To our knowledge, analyses quantifying associations between health interventions and U5M at a sub-national scale have not been carried out. Sub-national estimates of the geographical distribution of the effects of health interventions can guide control programmes to choose and implement most important interventions at a local scale.

The aim of the present study was to estimate geographically varying associations of health interventions (i.e., control interventions against malaria, water, sanitation and hygiene (WASH), reproductive health, breastfeeding, vaccinations, micronutrient supplementation and treatments) with U5M at the national and sub-national scale to identify interventions associated with the largest reduction in mortality at a sub-national scale, and to estimate hotspots of the U5M in Uganda. Bayesian geostatistical Weibull proportional hazards models were applied to DHS data and spatially varying covariates were introduced to assess the effects of interventions at local scale. The models were adjusted for socio-demographic, environmental and climatic factors. Bayesian kriging was used to estimate hotspots of U5M. Results assist control programmes to implement locally adapted interventions, and therefore reduce regional variation in all-cause U5M.

\section{Methods \\ Country profile}

Uganda is situated across the equator in East Africa. The country is bordered by the Democratic Republic of the Congo in the West, Kenya in the East, Rwanda in the South-West, Tanzania in the South and Sudan in the North. Uganda is a land-locked country with a surface area of $241,000 \mathrm{Km}^{2}$. The country is divided into 15 regions, which are further partitioned into 116 districts. The population is approximately 44 million people; about half of the population are younger than 15 years, while children below the age of 5 account for approximately $20 \%$ [10].

\section{Design and study setting}

All-cause child mortality data were obtained from women's birth histories, available in the 2011 DHS, which was carried out between May and December, 2011. A representative sample of 10,086 households was selected for the 2011 DHS, using a stratified two-stage cluster design. In the first stage, 404 clusters were selected from a list of clusters for the 2009/2010 Uganda National Household Survey. The second stage involved selecting households from a complete listing of households in each cluster. Overall, 8674 women aged 15-49 years who were either permanent residents of the households or visitors who slept in the households the night 
before the survey were eligible to be interviewed on characteristics regarding their children. Mortality data were collected on 7878 children representing the number of children born in the period of 5 years preceding the date of the survey.

\section{Data and sources \\ Health interventions}

The The DHS captures data relating to a number of health interventions, including malaria, micronutrients intake and treatments, the latter depending on whether drugs were taken in the previous night of the survey, 7 days, 2 weeks or 6 months prior to the survey. Such coverages may not reflect the extent of intervention utilization in the 5 years preceding the survey. Thus, to obtain representative estimates of intervention coverages for the period of 5 years preceding the 2011 DHS, we averaged health intervention coverages of the 2006 and 2011 DHS. The 2006 DHS collected data on malaria control interventions different from those in the 2011 DHS, that is, households with at least one insecticidetreated net (ITN), U5 sleeping under an ITN and indoor residual spraying (IRS). For consistency, interventions of the 2009 Uganda Malaria Indicator Survey (MIS) were utilized since they matched with those in the 2011 DHS.

Health interventions considered in this paper comprise of malaria, WASH practices, reproductive health, breastfeeding, vaccinations, micronutrients supplementation and treatments of diseases. Coverage of health interventions was generated at the cluster level [7] because data on various interventions such as the vaccination status of dead children are not reported at an individual level in the DHS. Data at clusters were used to obtain intervention coverages at regions.

Data on malaria interventions were collected by means of household questionnaires and included use and ownership of ITN and IRS. Standard guidelines of the Roll Back Malaria (RBM) were followed in the generation of malaria intervention coverage indicators [11]. The ITN use indicators derived in this analysis comprised the percentage of children U5 and the percentage of the population who slept under an ITN the night preceding the survey and the percentage of ITN used by the population in a household the previous night. The indicator on IRS coverage was generated as the percentage of households sprayed in the past 12 months. ITN ownership indicators included the percentage of households with at least one ITN, the percentage of households with one ITN for every two people and the percentage of the population with access to an ITN within their household. WASH interventions included the percentage of households with an improved source of drinking water, the percentage of households with improved sanitation facilities and the percentage of households with both water and soap/detergent at hand washing places.

Data on the coverage of reproductive health, breastfeeding, vaccinations, micronutrients supplementation and treatment interventions were collected from all eligible women using a pretested questionnaire. The questionnaire comprised reproductive health interventions (the percentage of married women using any family planning method, percentage of pregnant mothers receiving antenatal care (ANC) from a skilled provider, the percentage of pregnant women making four or more ANC visits during their entire pregnancy, the percentage of women who received intermittent preventive treatment for malaria during pregnancy (IPTp), the percentage of births that took place with the assistance of a skilled provider and the percentage of newborns receiving first postnatal checkup from a skilled provider within 2 days after delivery, breastfeeding (the percentage of infants who started breastfeeding within $1 \mathrm{~h}$ of birth and the percentage of infants exclusively breastfed during the first 6 months after birth), vaccinations (the percentage of the last-born child fully protected against neonatal tetanus, the percentage of children vaccinated with BCG and measles, the percentage of children with complete vaccination of DPT and polio), micronutrients supplementation (the percentage of children receiving vitamin A supplements, the percentage of children receiving iron supplements in the past 7 days and the percentage of children living in households with iodized of salt) and treatments of diseases (the percentage of children with symptoms of acute respiratory infections (ARIs) who took antibiotics, the percentage of children with diarrhoea given fluid from oral rehydration solution (ORS) sachets or recommended home fluids (RHF), the percentage of children with diarrhoea given zinc sulphates, the percentage of children with fever during the 2 weeks prior to the survey and took ACT and those dewormed in the past 6 months).

Interventions with coverage $\geq 95 \%$ and those lacking sufficient coverage $(<5 \%)$ within the regions were excluded from the analysis due to lack of variation in estimating their relation with mortality. These were the percentage of households sprayed with IRS in the past 12 months (\%H_IRS, 7\%), the percentage of pregnant mothers receiving ANC from a skilled provider (ANC provider, 95\%), the percentage of children living in households with iodized salt (iodized salt; 99\%) and the percentage of children with diarrhoea given zinc sulphates (zinc; $2 \%$ ). Table 1 provides a list of health interventions assessed in the study.

\section{Environmental and climatic factors}

Environmental and climatic factors were obtained from remote sensing sources and aggregated at the cluster 
Table 1 Health interventions, Uganda DHS 2006, 2009 and 2011

\begin{tabular}{|c|c|}
\hline Intervention & Description of the intervention \\
\hline \multicolumn{2}{|l|}{ Malaria } \\
\hline$\% \mathrm{H} \_\mathrm{IRS}$ & $\begin{array}{l}\text { Percentage of households sprayed with Indoor } \\
\text { Residual Spraying (IRS) in the past } 12 \text { months }\end{array}$ \\
\hline$\% \mathrm{H}_{-} 1 \mathrm{ITN}$ & Percentage of households with atleast one ITN \\
\hline$\% \mathrm{H}_{-} 1 \mathrm{ITN} 2$ & $\begin{array}{l}\text { Percentage of households with atleast one ITN } \\
\text { for every two people }\end{array}$ \\
\hline \%P_ITNA & $\begin{array}{l}\text { Percentage of population with access to an ITN } \\
\text { within their household (Percentage of the } \\
\text { population that could sleep under an ITN, if each } \\
\text { ITN in the household were used by up to two } \\
\text { people) }\end{array}$ \\
\hline \%P_ITNS & $\begin{array}{l}\text { Percentage of the population in a household that } \\
\text { slept under an ITN the previous night of the } \\
\text { survey }\end{array}$ \\
\hline \%P_ITN5 & $\begin{array}{l}\text { Percentage of children under } 5 \text { years in a } \\
\text { household who slept under an ITN the previous } \\
\text { night of the survey }\end{array}$ \\
\hline$\% P_{-} I T N U$ & $\begin{array}{l}\text { Percentage of existing ITNs used by the populatio } \\
\text { in a household the previous night of the survey }\end{array}$ \\
\hline \multicolumn{2}{|l|}{ WASH } \\
\hline $\begin{array}{l}\text { Improved } \\
\text { water }\end{array}$ & $\begin{array}{l}\text { Percentage of households with improved source } \\
\text { of drinking water }\end{array}$ \\
\hline $\begin{array}{l}\text { Improved } \\
\text { sanitation }\end{array}$ & $\begin{array}{l}\text { Percentage of households using improved } \\
\text { sanitation facilities }\end{array}$ \\
\hline P_wsoap & $\begin{array}{l}\text { Percentage of households with soap or detergent } \\
\text { and water at hand washing place }\end{array}$ \\
\hline
\end{tabular}

Reproductive health

Family

planning

ANC provider

4+ ANC visits

IPTp

Skilled

delivery

Postnatal care

Within $1 \mathrm{~h}$
Exclusive
Vaccinations
Tetanus
toxoid
BCG
DPT
Polio

Breastfeeding

Percentage of married women using any family planning method

Percentage of pregnant mothers receiving ANC from a skilled provider

Percentage of pregnant women making four or more ANC visits during their entire pregnancy

Percentage of women who received intermittent preventive treatment for malaria during pregnancy

Percentage of births that took place with the assistance of a skilled provider

Percentage of newborns receiving first postnatal checkup from a skilled provider within 2 days after delivery

Percentage of infants who started breastfeeding within $1 \mathrm{~h}$ of birth

Percentage of infants exclusively breastfed during the first 6 months after birth

Percentage of last-born child fully protected against neonatal tetanus

Percentage of children vaccinated against BCG

Percentage of children with complete vaccination of DPT

Percentage of children with complete vaccination of polio
Table 1 Health interventions, Uganda DHS 2006, 2009 and 2011 (Continued)

\begin{tabular}{cl}
\hline Intervention & Description of the intervention \\
\hline Micronutrients & Percentage of children vaccinated against measles \\
VitaminA_sup & $\begin{array}{l}\text { Percentage of children receiving vitamin A } \\
\text { supplements in the past 6 months }\end{array}$ \\
Iron_sup & $\begin{array}{l}\text { Percentage of children receiving iron supplements } \\
\text { in the past 7 days }\end{array}$ \\
lodized salt & $\begin{array}{l}\text { Percentage of children living in households with } \\
\text { iodized of salt }\end{array}$ \\
Treatments & $\begin{array}{l}\text { Percentage of children with ARIs symptoms who } \\
\text { Antibiotics }\end{array}$ \\
ORS or RHF & $\begin{array}{l}\text { Percentage of children with diarrhoea given fluid } \\
\text { from oral rehydration solution (ORS) sachets or } \\
\text { recommended home fluids (RHF) }\end{array}$ \\
Zinc & $\begin{array}{l}\text { Percentage of children with diarrhoea given zinc } \\
\text { sulphates }\end{array}$ \\
ACT & $\begin{array}{l}\text { Percentage of children with fever during the 2 } \\
\text { weeks prior to the survey and took artemisinin- } \\
\text { combination therapy (ACT) } \\
\text { Percentage of children given deworming } \\
\text { medication in the past 6 months }\end{array}$ \\
\hline
\end{tabular}

level. Temporal predictors such as land surface temperature (LST), rainfall and normalized difference vegetation index (NDVI) were averaged for the entire year of 2011. Land cover types were provided in 17 categories according to the International Global Biosphere Programme (IGBP) classification scheme and re-grouped into three categories, that is, urban, forest and crops. Distance to permanent water bodies was calculated based on the water category of the land cover data. Table 2 contains a list of environmental and climatic

Table 2 Remote sensing data sources ${ }^{a}$

\begin{tabular}{llll}
\hline Source & Data type & $\begin{array}{l}\text { Temporal } \\
\text { resolution }\end{array}$ & $\begin{array}{l}\text { Spatial } \\
\text { resolution }\end{array}$ \\
\hline MODIS/Terra ${ }^{b}$ & LST $^{\top}$ & 8 days & $1 \mathrm{~km}$ \\
MODIS/Terra & NDVI $^{\mathrm{m}}$ & 16 days & $1 \mathrm{~km}$ \\
$\begin{array}{l}\text { U.S. Geological Survey-Earth } \\
\text { Resources Observation Systems } \\
\text { (USGSS) }\end{array}$ & Rainfall & 10 days & $8 \times 8 \mathrm{~km}^{2}$ \\
$\begin{array}{l}\text { Shuttle Radar Topographic } \\
\text { Mission (SRTM) }\end{array}$ & Altitude & na & $1 \times 1 \mathrm{~km}^{2}$ \\
$\begin{array}{l}\text { MODIS,IBGD type } \\
\text { Global Rural and Urban }\end{array}$ & $\begin{array}{l}\text { Land cover } \\
\text { Water bodies }\end{array}$ & na & $0.5 \times 0.5 \mathrm{~km}^{2}$ \\
\hline
\end{tabular}

na Not applicable; Land cover groups (forest, crops, urban); ${ }^{2}$ Land cover data accessed in June 2011 and other data accessed in November 2013; ${ }^{b}$ Moderate Resolution Imaging Spectroradiometer (MODIS)/Terra, available at: http:// modis.gsfc.nasa.gov/; 'Land surface temperature (LST) day and night;

${ }^{m}$ Normalized difference vegetation index 
factors together with their spatio-temporal resolutions and data sources.

\section{Demographic and socioeconomic factors}

Demographic and socioeconomic proxies, including maternal (education, literacy, residence, age at birth, early pregnancy termination, number of children born and working status) and child (sex, birth order, birth interval and mode of delivery) characteristics were incorporated in the analysis at an individual level and were captured using a household questionnaire. The household asset score was aggregated at the cluster level and considered in the analysis as a socioeconomic proxy for households' socioeconomic status.

\section{Statistical analysis}

A Bayesian geostatistical proportional hazards model assuming a baseline Weibull hazard function was fitted to quantify the associations between health interventions' coverage and U5M, and to identify the most important interventions. The models were fitted to child-specific deaths and censoring times. Environmental, climatic, demographic and socioeconomic factors were included in the model as potential confounders. Spatial correlation between clusters was modelled by a Gaussian process with a covariance matrix measuring correlation between any pair of clusters by an exponential function of the distance between them. Our model assumed that the relation between health interventions and mortality varied across regions by including spatially varying coefficients to capture the interventions effect. Spatial dependence in the interventions' effects was modelled by region-specific random effects assuming conditional autoregressive prior distributions.

To identify the most important interventions and characteristics associated with the U5M, Bayesian geostatistical variable selection was used, adopting a stochastic search approach. The selection consisted of introducing a binary indicator parameter for each of socio-demographic, IRS and land cover variables with values defining the covariate-specific inclusion probability in the model. We assumed that the indicator arises from a Bernoulli prior distribution with probability defining the variable-specific inclusion probability in the model. We have chosen a spike and slab prior for the regression coefficients, which is a mixture of normals with mixing proportion equal to the inclusion probability. The spike component shrinks the regression coefficient to zero when the variable is excluded and the slab assumes a non-informative normal prior distribution when the variable has high inclusion probability (i.e., $\geq 50 \%$ ). Environmental and climatic indicators (LST, NDVI, distance to permanent bodies and rainfall) were included or excluded in the model in a linear or categorical form. We introduced indicators with a multinomial prior distribution with three parameters corresponding to the probabilities of exclusion of a variable, inclusion in linear or categorical form. ITN coverage indicators were highly correlated with more than $85 \%$. Hence, only one (or none) ITN indicator among those measuring ownership and one (or none) ITN indicator among those defining use was selected. The ITN indicator with the highest probability of inclusion in each category was included in the final model. Health intervention indicators were standardized and a separate model adjusting for possible confounders was fitted for each selected intervention.

Maps were generated using ArcGIS version 10.5 (ESRI; Redlands, CA, USA). Descriptive data analysis was carried out in STATA version 14.0 (Stata Corporation; College Station, TX, USA). Bayesian variable selection and model fit were implemented in OpenBUGS 3.2.3 (Imperial College and Medical Research Council; London, UK). The effects of health interventions on U5M were summarized by posterior medians of their hazard rate ratios (HRR) and the corresponding 95\% Bayesian credible intervals (BCI). An estimate is considered statistically significant if its $95 \%$ BCI excludes one. Details on the Bayesian geostatistical methods are provided in the Additional file 1.

\section{Results}

Table 3 provides a summary of the U5M estimates and the coverage of health interventions at regional and country levels.

The overall U5M was 90 deaths per 1000 live births. There were large regional variations in mortality rates with the lowest (56 deaths per 1000 live births) in Kampala and the highest (152 deaths per 1000 live births) in the North-East. The discrepancies in U5M across regions suggest that mortality rates may be influenced by region-specific factors.

IRS is the malaria intervention with the lowest coverage (7\%). Among the WASH practices, the percentage of households having improved sanitation facilities was lowest (14\%). Postnatal care was the least implemented reproductive health intervention with $11 \%$ of the newborns receiving the intervention. Among vaccinations, BCG had the highest coverage (93\%). Almost all children (98\%) lived in households that use iodized salt. Iron supplementation coverage was the lowest micronutrient intake nationally $(7 \%)$ whereas zinc was the least implemented treatment (2\%).

Table 4 presents results from the Bayesian geostatistical variable selection. Variables selected with $50 \%$ or higher inclusion probabilities were incorporated into the 
Table 3 Coverage of interventions (\%) and U5MR at the regional and country levels, Uganda DHS 2006 and 2011

\begin{tabular}{|c|c|c|c|c|c|c|c|c|c|c|c|}
\hline Interventions & Central1 & Central2 & Kampala & $\begin{array}{l}\text { East- } \\
\text { Central }\end{array}$ & $\begin{array}{l}\text { Mid- } \\
\text { Eastern }\end{array}$ & $\begin{array}{l}\text { North- } \\
\text { East }\end{array}$ & $\begin{array}{l}\text { Mid- } \\
\text { North }\end{array}$ & $\begin{array}{l}\text { West- } \\
\text { Nile }\end{array}$ & $\begin{array}{l}\text { Mid- } \\
\text { Western }\end{array}$ & $\begin{array}{l}\text { South- } \\
\text { West }\end{array}$ & Country \\
\hline \multicolumn{12}{|l|}{ Malaria } \\
\hline$\% \mathrm{H} \_I \mathrm{RS}$ & 1 & 3 & 5 & 1 & 2 & 2 & 49 & 1 & 0 & 1 & 7 \\
\hline$\% \mathrm{H}_{\text {_llTN }}$ & 47 & 42 & 54 & 36 & 58 & 67 & 65 & 67 & 51 & 51 & 54 \\
\hline \%H_1ITN2 & 24 & 21 & 37 & 11 & 19 & 27 & 23 & 25 & 21 & 20 & 22 \\
\hline \%P_ITNA & 37 & 33 & 47 & 23 & 38 & 47 & 45 & 47 & 38 & 37 & 39 \\
\hline \%P_ITNS & 27 & 24 & 40 & 19 & 33 & 45 & 34 & 40 & 29 & 26 & 31 \\
\hline \%P_ITN5 & 47 & 43 & 62 & 36 & 50 & 58 & 53 & 60 & 50 & 45 & 38 \\
\hline \%P_ITNU & 27 & 24 & 40 & 19 & 33 & 45 & 35 & 41 & 28 & 26 & 31 \\
\hline \multicolumn{12}{|l|}{ WASH } \\
\hline Improved water & 45 & 69 & 90 & 78 & 83 & 85 & 76 & 71 & 58 & 45 & 69 \\
\hline Improved sanitation & 25 & 25 & 17 & 15 & 9 & 3 & 5 & 4 & 9 & 7 & 14 \\
\hline P_wsoap & 45 & 27 & 42 & 12 & 9 & 2 & 10 & 5 & 32 & 16 & 27 \\
\hline \multicolumn{12}{|l|}{ Reproductive health } \\
\hline Family planning & 35 & 35 & 48 & 28 & 23 & 8 & 17 & 14 & 27 & 28 & 27 \\
\hline ANC provider & 89 & 94 & 97 & 92 & 95 & 97 & 96 & 98 & 95 & 95 & 95 \\
\hline 4+ ANC visits & 52 & 49 & 64 & 44 & 39 & 56 & 48 & 62 & 46 & 45 & 48 \\
\hline IPTp & 18 & 19 & 23 & 15 & 26 & 29 & 19 & 18 & 27 & 24 & 23 \\
\hline Skilled delivery & 57 & 60 & 91 & 61 & 47 & 31 & 42 & 47 & 44 & 37 & 50 \\
\hline Postnatal care & 11 & 8 & 29 & 8 & 14 & 19 & 13 & 11 & 9 & 1 & 11 \\
\hline \multicolumn{12}{|l|}{ Breastfeeding } \\
\hline Within $1 \mathrm{~h}$ & 42 & 52 & 56 & 55 & 41 & 70 & 40 & 38 & 55 & 45 & 48 \\
\hline Exclusive & 56 & 64 & 65 & 53 & 58 & 52 & 58 & 57 & 56 & 48 & 52 \\
\hline \multicolumn{12}{|l|}{ Vaccinations } \\
\hline Tetanus toxoid & 71 & 80 & 80 & 80 & 81 & 93 & 82 & 85 & 79 & 80 & 80 \\
\hline $\mathrm{BCG}$ & 81 & 92 & 93 & 92 & 97 & 100 & 95 & 98 & 93 & 86 & 93 \\
\hline DPT & 59 & 63 & 71 & 57 & 70 & 90 & 70 & 72 & 74 & 70 & 68 \\
\hline Polio & 51 & 57 & 64 & 54 & 62 & 65 & 58 & 61 & 69 & 71 & 61 \\
\hline Measles & 67 & 69 & 77 & 65 & 70 & 91 & 76 & 71 & 79 & 69 & 72 \\
\hline \multicolumn{12}{|l|}{ Micronutrients } \\
\hline VitaminA_sup & 30 & 36 & 41 & 56 & 50 & 74 & 54 & 42 & 55 & 37 & 47 \\
\hline Iron_sup & 4 & 4 & 3 & 5 & 8 & 12 & 12 & 9 & 5 & 3 & 7 \\
\hline lodized salt & 99 & 98 & 100 & 98 & 99 & 100 & 99 & 96 & 94 & 96 & 98 \\
\hline \multicolumn{12}{|l|}{ Treatments } \\
\hline Antibiotics & 55 & 49 & 72 & 33 & 46 & 30 & 43 & 54 & 58 & 42 & 47 \\
\hline ORS or RHF & 44 & 52 & 48 & 49 & 44 & 77 & 53 & 46 & 37 & 28 & 46 \\
\hline Zinc & 1 & 2 & 2 & 2 & 1 & 1 & 2 & 3 & 3 & 0 & 2 \\
\hline $\mathrm{ACT}$ & 39 & 34 & 42 & 34 & 31 & 81 & 43 & 40 & 37 & 30 & 36 \\
\hline Deworming & 47 & 45 & 58 & 40 & 48 & 65 & 44 & 38 & 51 & 41 & 46 \\
\hline \multicolumn{12}{|l|}{ Mortality rates } \\
\hline U5MR & 83 & 79 & 56 & 104 & 80 & 152 & 76 & 100 & 95 & 99 & 90 \\
\hline
\end{tabular}

final model (e.g., improved source of drinking water and improved sanitation facilities from the WASH practices interventions).
Results presented in Table 5 show that at the national level all interventions except family planning were associated with a lower risk of U5M, with ACT associated 
Table 4 Posterior inclusion probabilities of interventions, socioeconomic, demographic and environmental/climatic factors

\begin{tabular}{|c|c|}
\hline Variable & Inclusion probability (\%) \\
\hline \multicolumn{2}{|l|}{ Malaria } \\
\hline \multicolumn{2}{|l|}{ ITN access } \\
\hline None & 8.4 \\
\hline$\% \mathrm{H}_{-} 1 \mathrm{ITN}$ & 27.2 \\
\hline$\% \mathrm{H}_{-} 1 \mathrm{ITN} 2$ & 4.0 \\
\hline \%P_ITNA & $60.4^{\mathrm{a}}$ \\
\hline \multicolumn{2}{|l|}{ ITN use } \\
\hline None & 3.1 \\
\hline \%P_ITNS & 19.1 \\
\hline \%P_ITN5 & 12.3 \\
\hline \%P_ITNU & $65.5^{\mathrm{a}}$ \\
\hline \multicolumn{2}{|l|}{ WASH } \\
\hline Improved water & $66.6^{\mathrm{a}}$ \\
\hline Improved sanitation & $52.2^{\mathrm{a}}$ \\
\hline P_wsoap & 23.8 \\
\hline \multicolumn{2}{|l|}{ Reproductive health } \\
\hline Family planning & $69.0^{\mathrm{a}}$ \\
\hline 4+ ANC visits & 25.0 \\
\hline IPTp & $60.8^{\mathrm{a}}$ \\
\hline Skilled delivery & $70.0^{\mathrm{a}}$ \\
\hline Postnatal care & $76.4^{\mathrm{a}}$ \\
\hline \multicolumn{2}{|l|}{ Breastfeeding } \\
\hline Within $1 \mathrm{~h}$ & $73.8^{\mathrm{a}}$ \\
\hline Exclusive & $100.0^{\mathrm{a}}$ \\
\hline \multicolumn{2}{|l|}{ Vaccinations } \\
\hline $\mathrm{BCG}$ & 22.0 \\
\hline DPT & $83.0^{\mathrm{a}}$ \\
\hline Polio & 14.8 \\
\hline Measles & $83.4^{\mathrm{a}}$ \\
\hline \multicolumn{2}{|l|}{ Micronutrients } \\
\hline VitaminA_sup & $60.0^{\mathrm{a}}$ \\
\hline Iron_sup & 38.6 \\
\hline \multicolumn{2}{|l|}{ Treatments } \\
\hline Antibiotics & $55.0^{\mathrm{a}}$ \\
\hline ORS or RHF & $57.0^{\mathrm{a}}$ \\
\hline ACTs & $61.0^{\mathrm{a}}$ \\
\hline Deworming & $81.0^{\mathrm{a}}$ \\
\hline \multicolumn{2}{|c|}{ Socio-economic and demographic } \\
\hline \multicolumn{2}{|l|}{ Child } \\
\hline Sex & $73.2^{\mathrm{a}}$ \\
\hline \multicolumn{2}{|l|}{ Maternal } \\
\hline Age at birth & $100.0^{\mathrm{a}}$ \\
\hline Number of children & $100.0^{\mathrm{a}}$ \\
\hline
\end{tabular}

Table 4 Posterior inclusion probabilities of interventions, socioeconomic, demographic and environmental/climatic factors (Continued)

\begin{tabular}{ll}
\hline Variable & Inclusion probability (\%) \\
\hline Education level & $70.2^{\mathrm{a}}$ \\
Residence (urban vs rural) & $59.6^{\mathrm{a}}$ \\
Working status & 34.0 \\
Household & \\
$\quad$ Age of head & 0.0 \\
$\quad$ Wealth index & $81.2^{\mathrm{a}}$ \\
Environmental/Climatic factors & \\
Land cover & 33.0 \\
LST day: None & 73.0 \\
LST day continuous & 27.0 \\
LST day categorical & 0.0 \\
LST night: None & 90.6 \\
LST night continuous & 8.1 \\
LST night categorical & 1.3 \\
NDVI: None & 41.2 \\
NDVI continuous & $58.8^{\mathrm{a}}$ \\
NDVI categorical & 0.0 \\
Rainfall: None & 80.3 \\
Rainfall continuous & 12.2 \\
Rainfall categorical & 8.5 \\
d_water: None & 100.0 \\
d_water continuous & 0.0 \\
d_water categorical & 0.0 \\
\hline
\end{tabular}

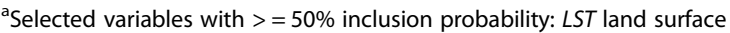
temperature, NDVI normalized difference vegetation index, $d \_$water distance to permanent water bodies

with a highest reduction $(\mathrm{HRR}=0.60$; 95\% BCI: 0.11 , 0.79 ).

Sub-national analysis (Table 5) indicates that in Central 2, Mid-Western and South-West regions, the largest reduction in the U5M burden was associated with ITN access. The intervention also had a large association on U5M in Central 1 and Kampala. Improved source of drinking water was associated with most U5M decrease in Mid-North and West-Nile. Improved source of drinking water was, in addition, associated with U5M in Central 2, East-Central, Kampala and Mid-Eastern. Improved sanitation facilities were associated with the highest decline in U5M in the North-East. The coverage of improved sanitation facilities also had an important association with mortality in South-West and West-Nile. In Kampala and Mid-Eastern, IPTp, had the largest association with U5M. The relation between IPTp and mortality was statistically important in Central 1 , MidWestern and West-Nile. In Central 1 and East-Central, ORS or RHF and postnatal care were respectively 


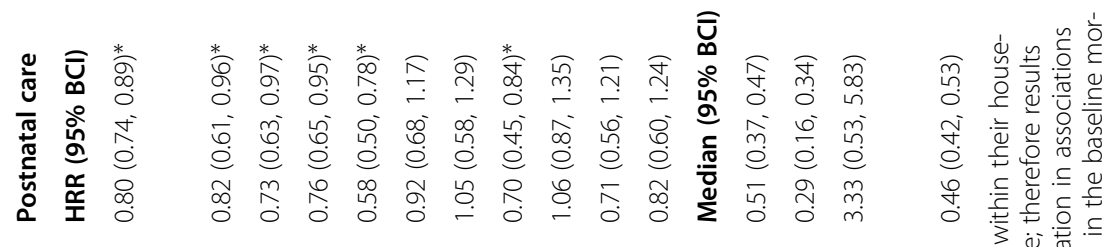

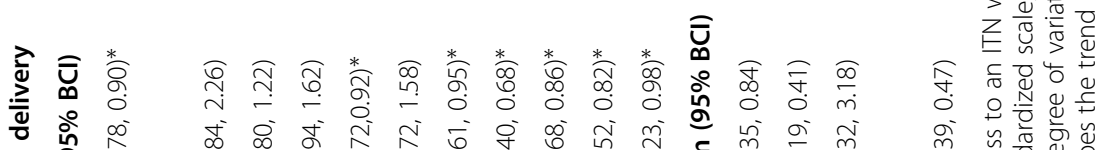

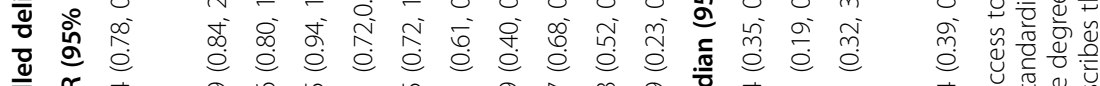

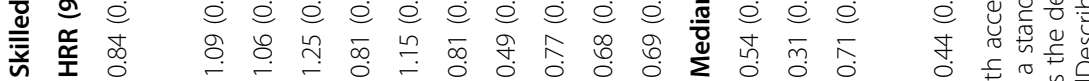

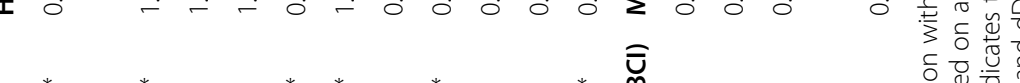

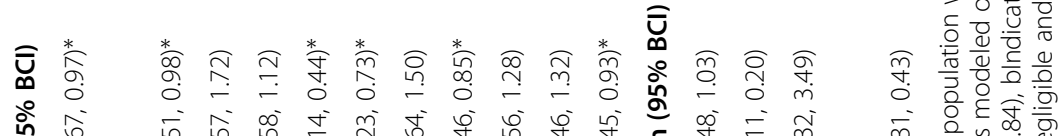

น̂n

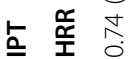

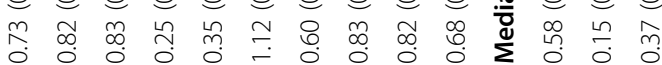

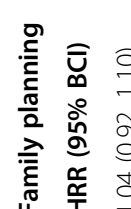

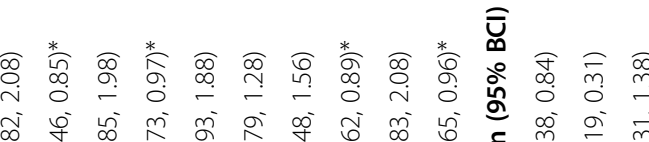

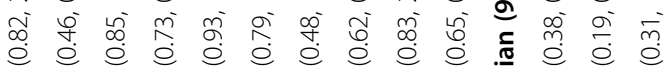

શิ

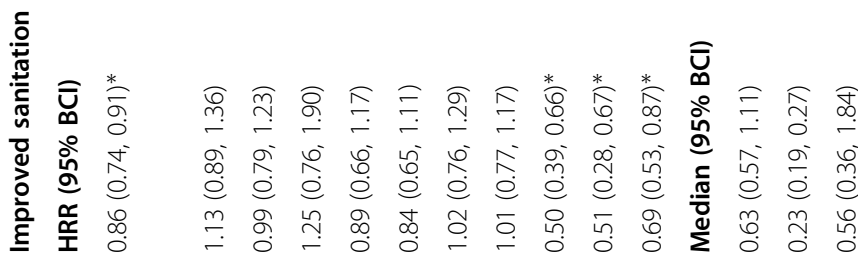

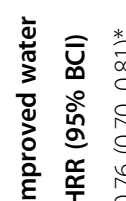

* * * * * *

जิ

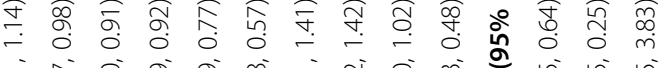

j.

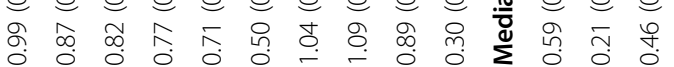

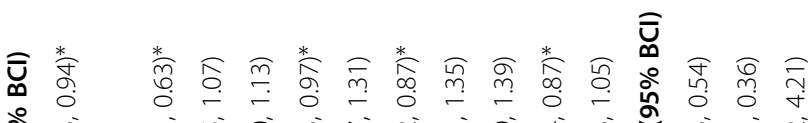

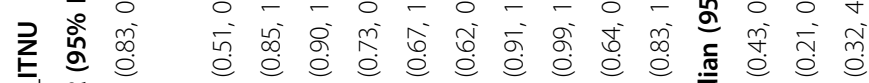

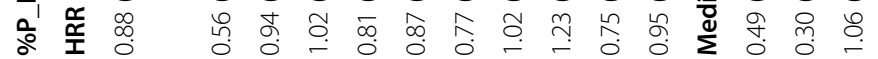

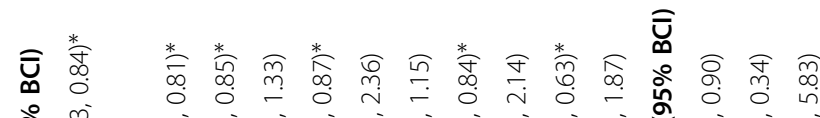

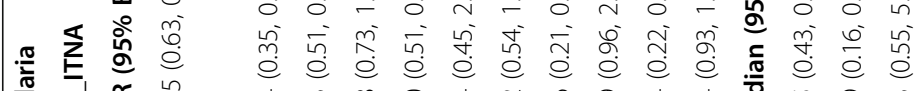

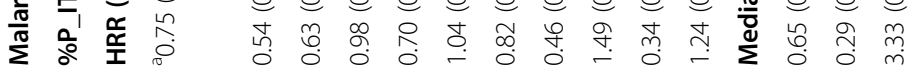

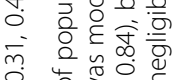

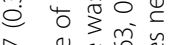

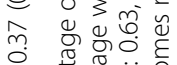

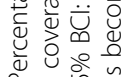

ô.

ते

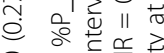

잉 음

진 त्रें

志主范

ส

o

กั

品 突

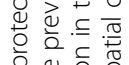

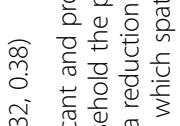

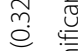

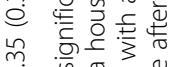

*

vi

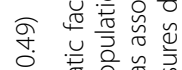

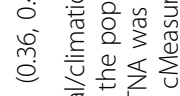

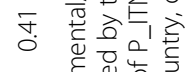

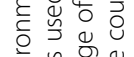

क्ष

의

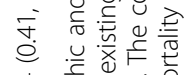

कृ
Ұ.

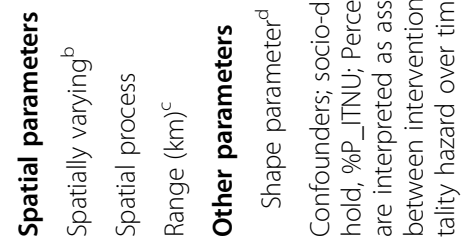

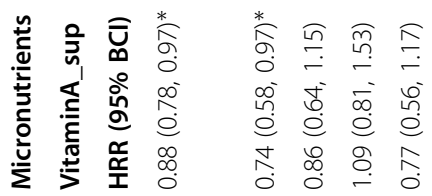

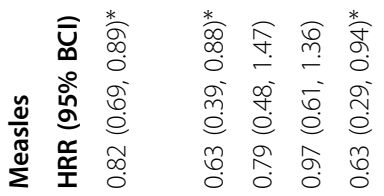

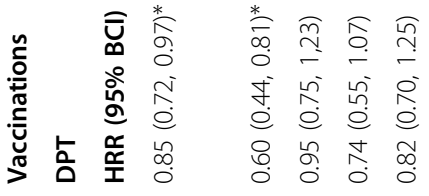

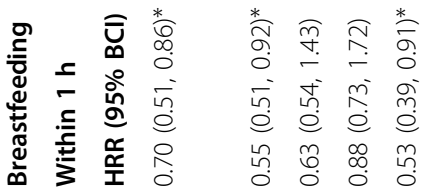

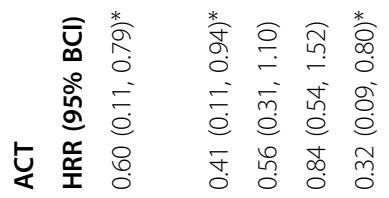

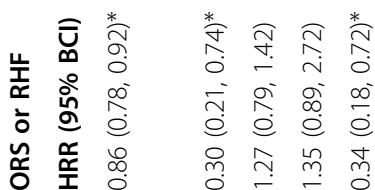

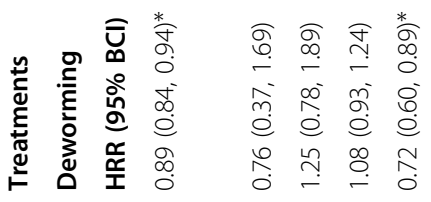

过 


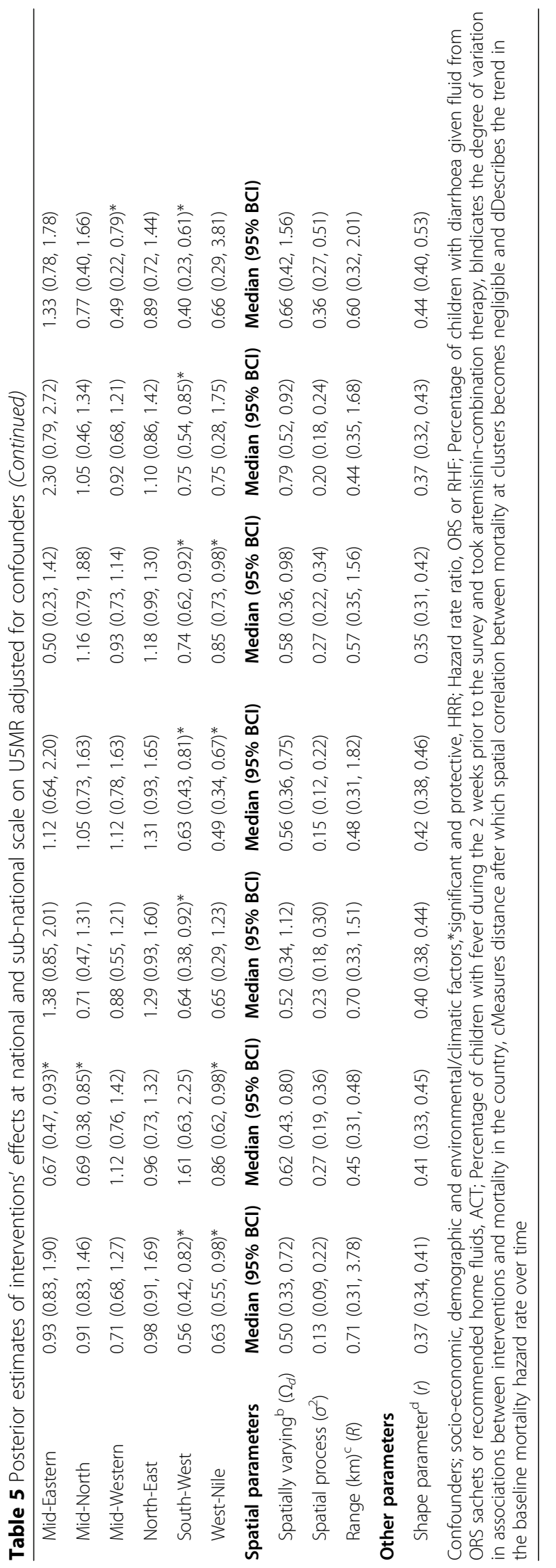




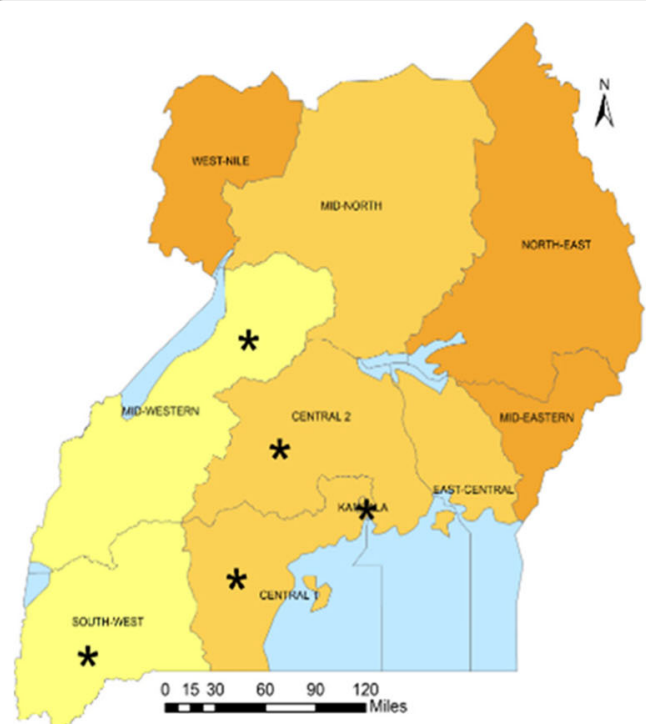

(a)

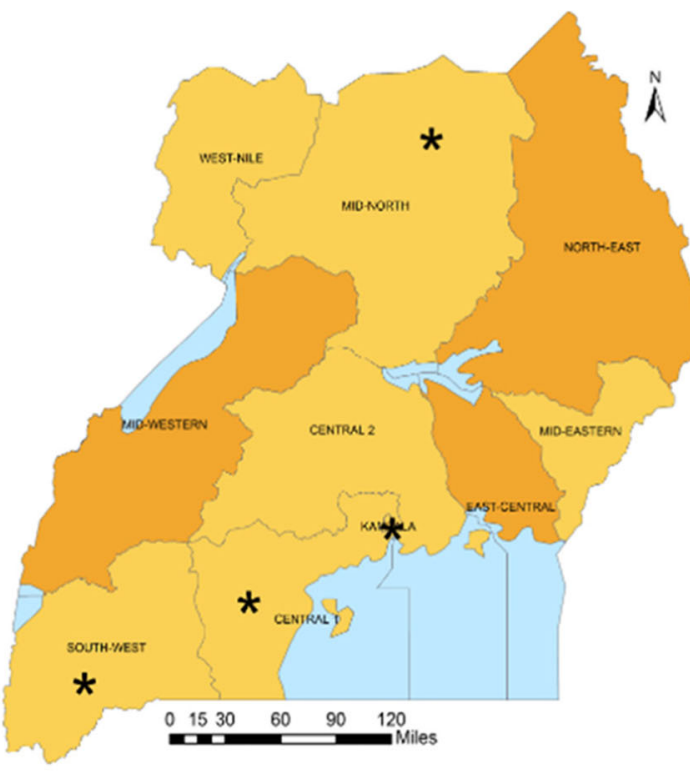

(b)
Effect of interventions (Hazard ratios)

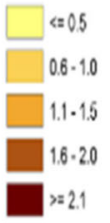

Fig. 1 Geographical distribution of the associations (Hazard rate ratios) of malaria interventions with under-five mortality; ( ${ }^{*}$ statistically significant association of interventions and protective against mortality); a Percentage of population with access to an ITN within their household, $\mathbf{b}$ Percentage of existing ITNs used by the population in a household the previous night of the survey

associated with the highest decreases in U5M. The coverage of postnatal had an important association on U5M in Central 1, Central 2, Kampala and Mid-Western. ORS or RHF was associated with lower mortality hazards in Kampala and South-West. Family planning had an important association on mortality in Central 2, Kampala, North-East and
West-Nile. Figures 1, 2, 3, 4 and 5 summarize graphically the spatially varying effects of all interventions on U5M.

Table 6 show that socio-demographic, environmental and climatic factors were important determinants of U5M. For instance, children born to mothers residing in urban areas had lower hazards of mortality relative to

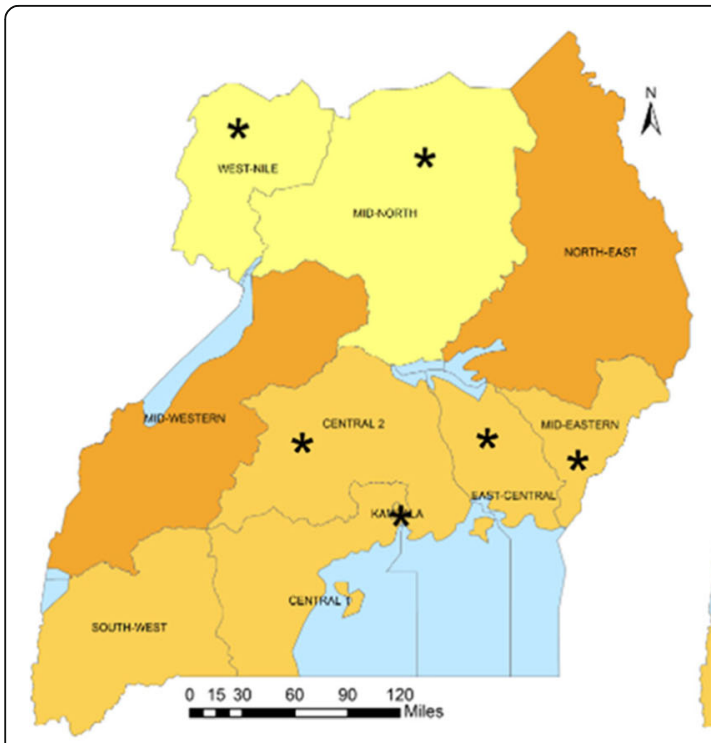

(a)

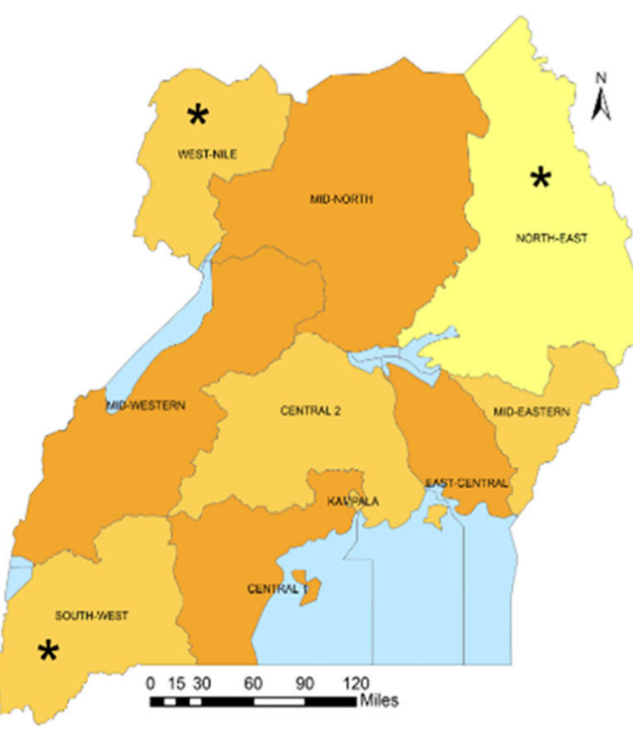

(b)
Effect of interventions (Hazard ratios)

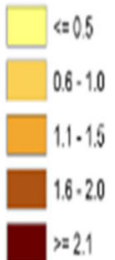

Fig. 2 Geographical distribution of the associations (Hazard rate ratios) of water, sanitation and hygiene practices with under-five mortality; (*statistically significant effect of interventions and protective against mortality); a Percentage of households with improved source of drinking water, $\mathbf{b}$ Percentage of households using improved sanitation facilities 


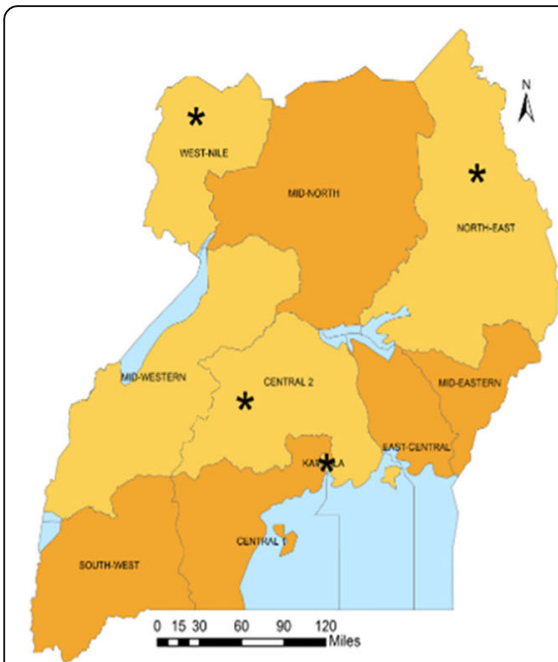

(a)

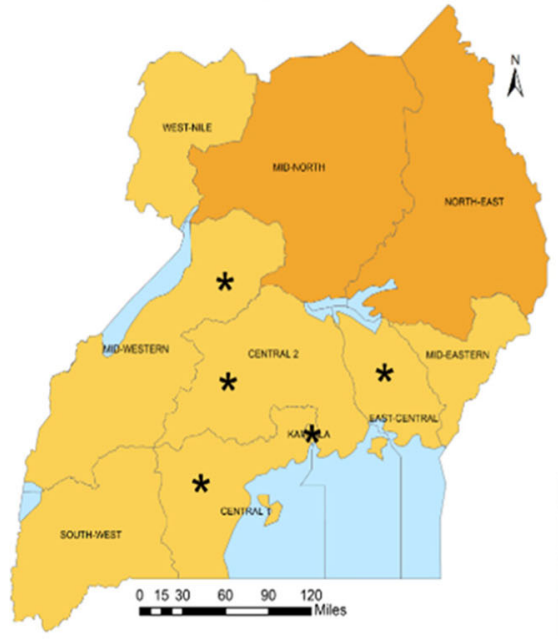

(d)

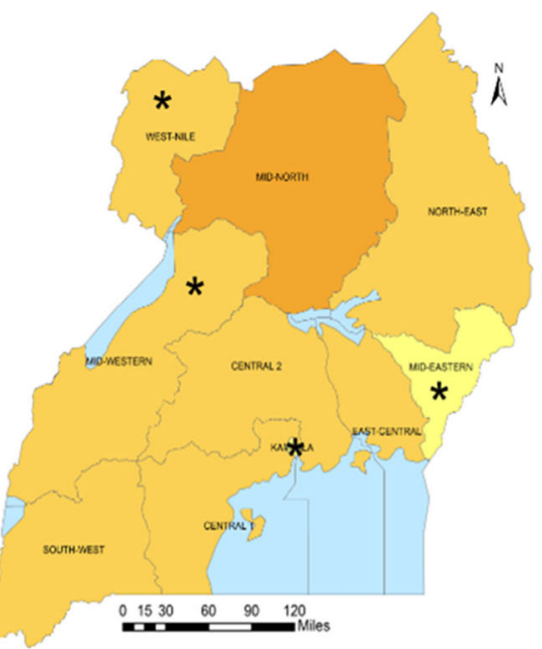

(b)

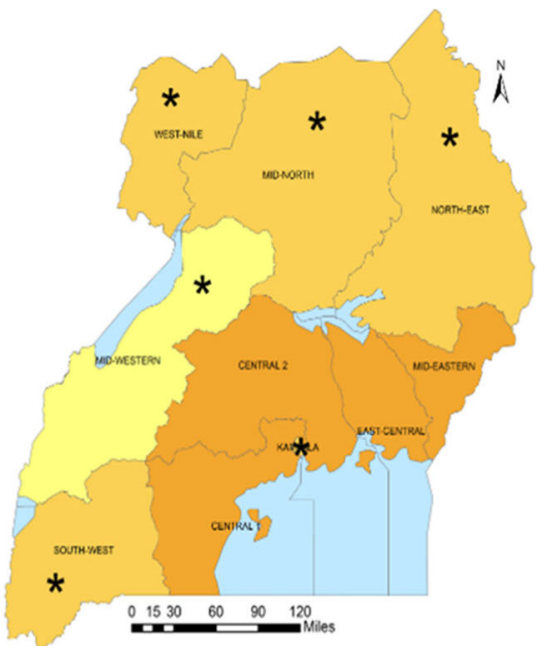

(c)

\section{Effect of interventions (Hazard ratios)}

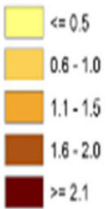

Fig. 3 Geographical distribution of the associations (Hazard rate ratios) of reproductive health interventions with under-five mortality; (*statistically significant association of interventions and protective against mortality); a Percentage of married women using any family planning method, $\mathbf{b}$ Percentage of women who received intermittent preventive treatment for malaria during pregnancy, c Percentage of births that took place with the assistance of a skilled provider, $\mathbf{d}$ Percentage of newborns receiving first postnatal checkup from a skilled provider within 2 days after delivery

those in rural areas. Environmental and climatic factors were associated with lower hazards. Children living in areas with a higher NDVI showed increased survival times.

\section{Discussion}

We quantified the associations of health interventions on all-cause U5M at national and sub-national scales in Uganda. The analysis took into account confounding effects of socio-demographic and environmental and climatic factors, which have been shown to be significantly associated with mortality [1-7]. We found strong geographical variations in the effects of health interventions on all-cause U5M across Uganda.
Findings at the national level indicated that ACT, initiation of breast feeding within $1 \mathrm{~h}$ of birth, IPTp, ITN access and improved source of drinking water were the health interventions associated with a highest reduction in U5M. However, these interventions were poorly implemented in the country with coverage below $50 \%$ yet the prevalences of diseases targeted by these interventions are high [8]. For example, malaria prevalence was at least $40 \%$ nationally and in $80 \%$ of the regions [9]. Other interventions, which were associated with a significant reduction in mortality U5M include ITN use, improved sanitation facilities, skilled delivery, postnatal care, complete DPT and measles vaccination, vitamin A supplementation, deworming in the past 6 months and ORS or RHF. These variables are among the essential 


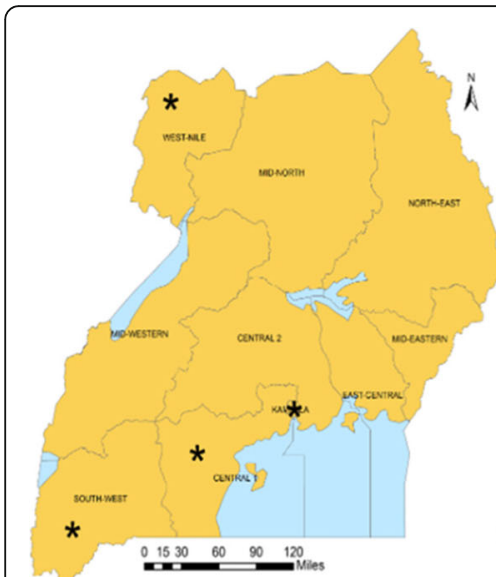

(a)

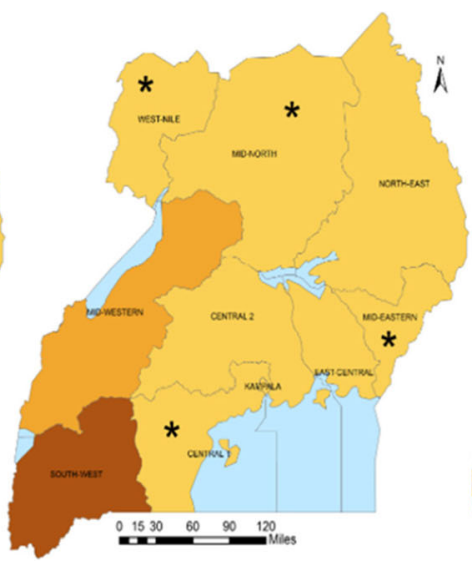

(b)

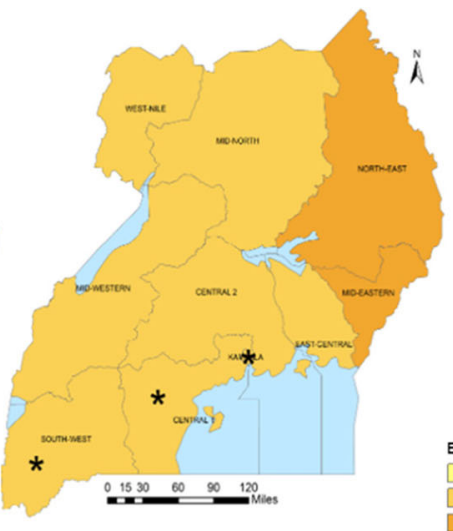

(c)

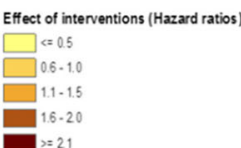

Fig. 4 Geographical distribution of the associations (Hazard rate ratios) of breastfeeding and vaccinations with under-five mortality; ( ${ }^{*}$ statistically significant association of interventions and protective against mortality); a Percentage of infants who started breastfeeding within $1 \mathrm{~h}$ of birth, $\mathbf{b}$ Percentage of children with complete vaccination of DPT, c Percentage of children vaccinated against measles

health interventions that have been associated with a decrease in child mortality in a review by Lassi et al. [10]. Our findings corroborate results reported from analyses of DHS data [11] and community-based studies in Uganda [12, 13]. Similar results were reported in other settings. For instance, Masanja et al. in Tanzania [14] analysed DHS data and found that increased coverage of key child-survival interventions, such as sleeping under ITNs, vitamin A supplementation, immunisation and exclusive breastfeeding accelerated progress in reducing U5M in Tanzania. In addition, analysis of cohort studies in Burkina Faso [15], randomised control trials in Guinea Bissau [16] and systematic reviews [17, 18] reported vaccinations to be associated with declines in child mortality. Our findings showed that DPT and measles vaccination were not statistically associated with U5M in Uganda although they had high coverage. This could be related to the untimely receipt of the vaccines, which might have hindered optimal immune response to the vaccines. According to guidelines developed by the World Health Organization (WHO) [19], children are considered fully vaccinated when they have received a vaccination against tuberculosis (BCG), three doses each of the diphtheria, pertussis and tetanus (DPT) and polio vaccines and a measles vaccination by the age of 12 months. At the time of the survey, only half of the children had received all basic vaccinations by the appropriate age of 12 months [8]. Untimely vaccinations contribute to coverage figures leading to an overestimation of actual population immunity [20-22].

The varying associations between interventions and U5M across regions could be explained by external factors, which this analysis has not taken into account but influence the effect of interventions, such as the health system, which differs between regions. This suggests for further efforts beyond increasing coverage of interventions, such as an approach which improves external factors as well as intervention coverage.

Study results confirmed earlier finding that household socioeconomic status is protective against U5M. The improved socioeconomic status boosts the effect of interventions despite their low coverages. Better socioeconomic status has been shown to reduce U5M [23-26]. In Central 2, Mid-Western and South-West, in which ITN access had the largest association with mortalityU5M, over $65 \%$ of households in the three regions fall either in the middle, fourth or highest wealth quintile [8]. More than $65 \%$ percent of households in EastCentral, in which postnatal care had a leading association with U5M, are in the middle, fourth or highest wealth quintile [8]. IPTp had a strongest association with mortality in Kampala and over $90 \%$ of households in this region are in the highest quintile [8]. In Central 1 where ORS or RHF had a highest association with U5M, over $65 \%$ of households either belong to the fourth or highest wealth quintile [8]. The improved socioeconomic status in these regions might have contributed to the success of the poorly implemented interventions. The NorthEast, in which coverage of several interventions was satisfactory (e.g., improved source of drinking water, DPT and measles vaccination, deworming, ORS or RHF and $\mathrm{ACT}$ ), over $80 \%$ of households in this region are in the lowest wealth quintile [8]. The high poverty level in the North-East could have hindered the effectiveness of many interventions, which were adequately implemented in this region.

This work comes with a number of limitations. First, data on several health interventions for dead children 


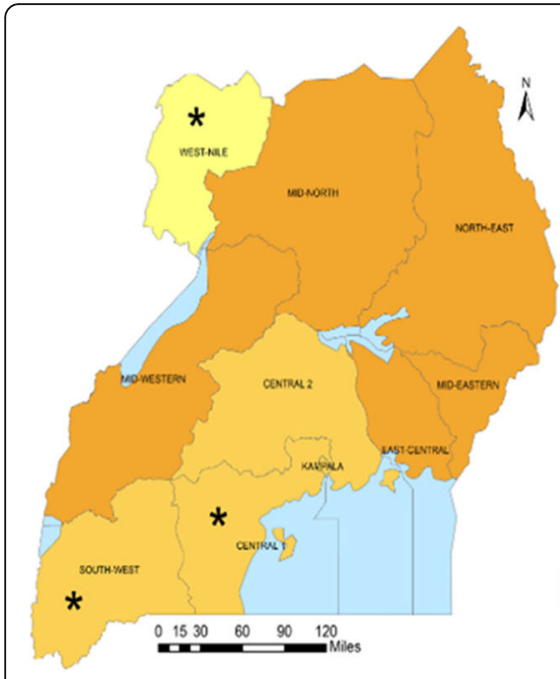

(a)

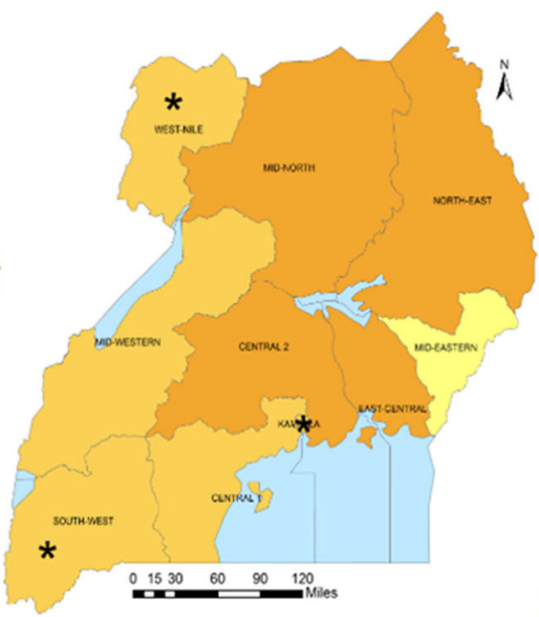

(b)

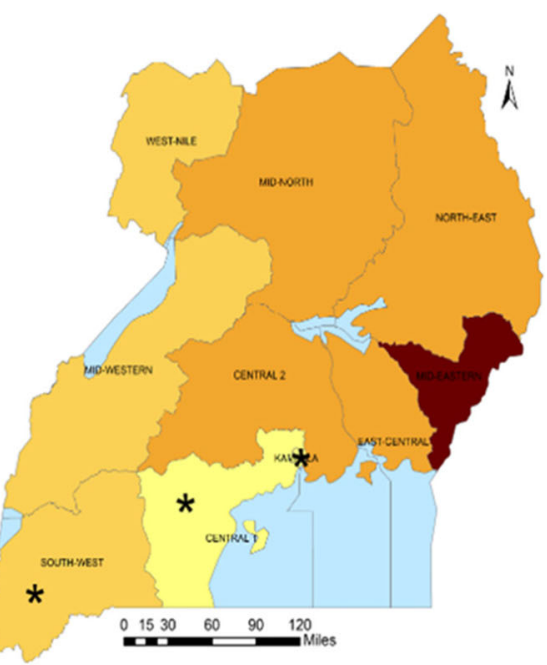

(c)

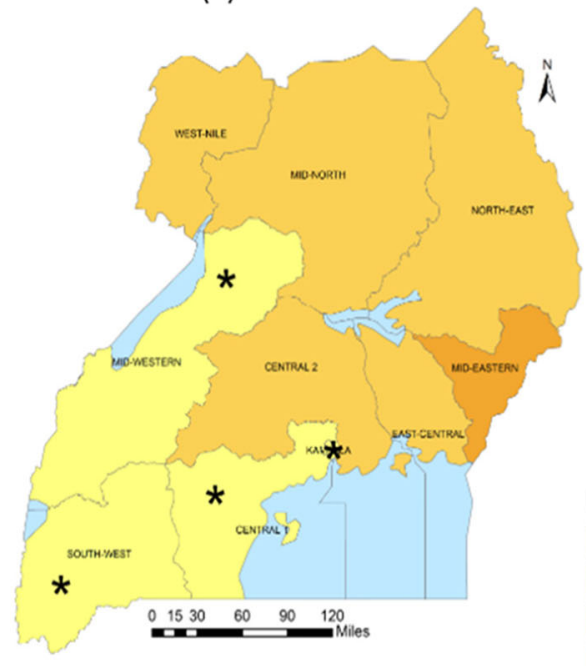

Effect of interventions (Hazard ratios)

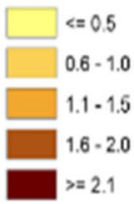

Fig. 5 Geographical distribution of the associations (Hazard rate ratios) of micronutrients intake and treatments with under-five mortality; ( ${ }^{*}$ statistically significant association of interventions and protective against mortality); a Percentage of children receiving vitamin A supplements in the past 6 months, $\mathbf{b}$ Percentage of children given deworming medication in the past 6 months, $\mathbf{c}$ Percentage of children with diarrhoea given fluid from oral rehydration solution sachets or recommended home fluids, $\mathbf{d}$ Percentage of children with fever during the 2 weeks prior to the survey and took artemisinin-combination therapy

could not be collected in the DHS. Hence, the intervention-mortality relation could not be estimated using interventions data at an individual level. Therefore, we created intervention coverage indicators measuring the proportion of children using an intervention at the cluster level. We linked individual U5M data to cluster intervention coverage and quantified associations between interventions and U5M while adjusting for sociodemographics and environmental/climatic factors.

Further, the analysis in this work was based on aggregations of the coverages of interventions at the cluster level, hence considering coverages of only living children. This could have resulted in a bias in associations due to the difference in the coverage of health interventions between the living and dead. This is because the association between coverage of interventions and mortality may differ between the living and dead children, and ignoring coverage of interventions for the dead children could induce selection bias in estimates of the coverage of interventions at a cluster level and also in estimates of associations between coverage of interventions and mortality [27]. Therefore, there is need to implement a correction to the observed coverage of interventions. Reniers et al. [28] proposed a correction based on the Bayes' theorem and an initial estimate of the relative risk of mortality for each intervention that 


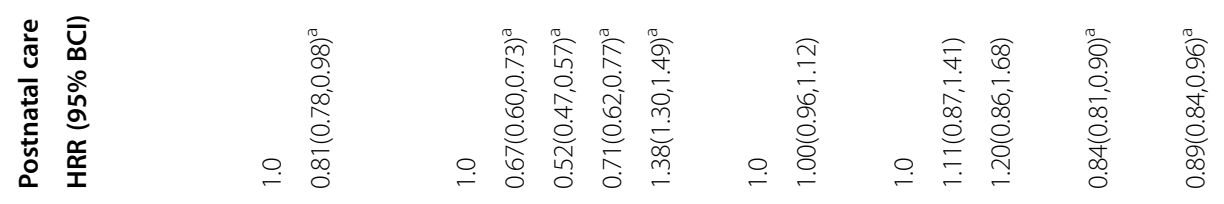

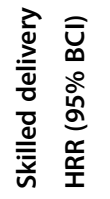
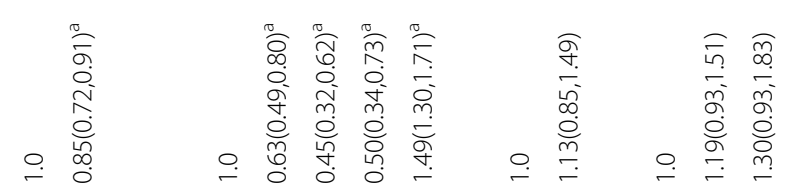

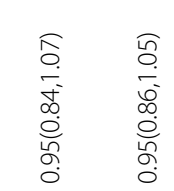

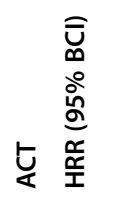
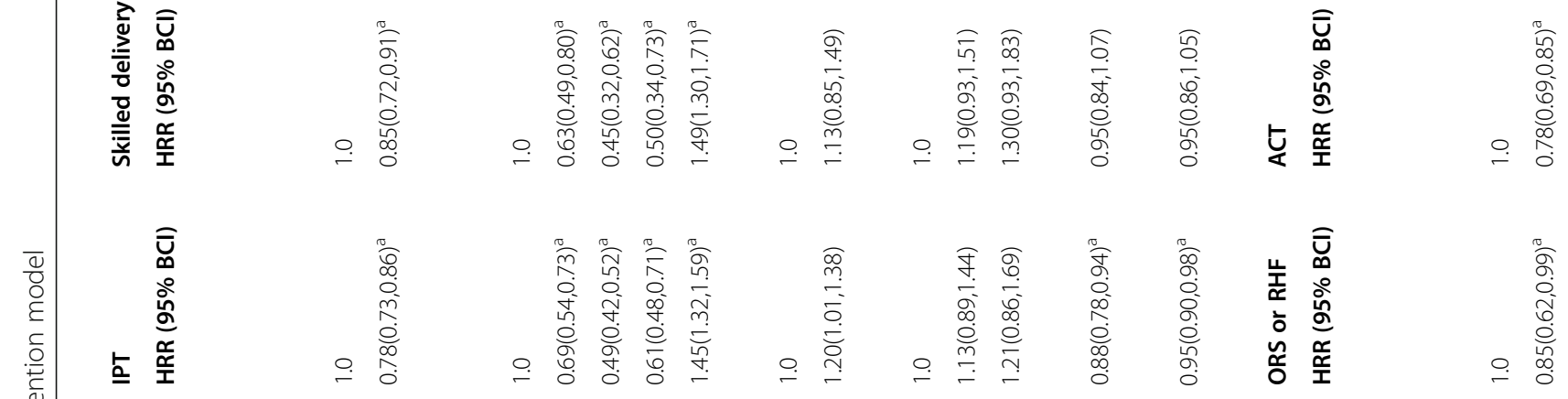

$\begin{array}{lll}0 & \\ 0 & 0 \\ 0 & 0 \\ 0 & 0 \\ 0 & 0 \\ 0 & 8 \\ 0 & 0 \\ 0 & 0 \\ 0 & 0 \\ 0 & 0 \\ 0 & 0\end{array}$
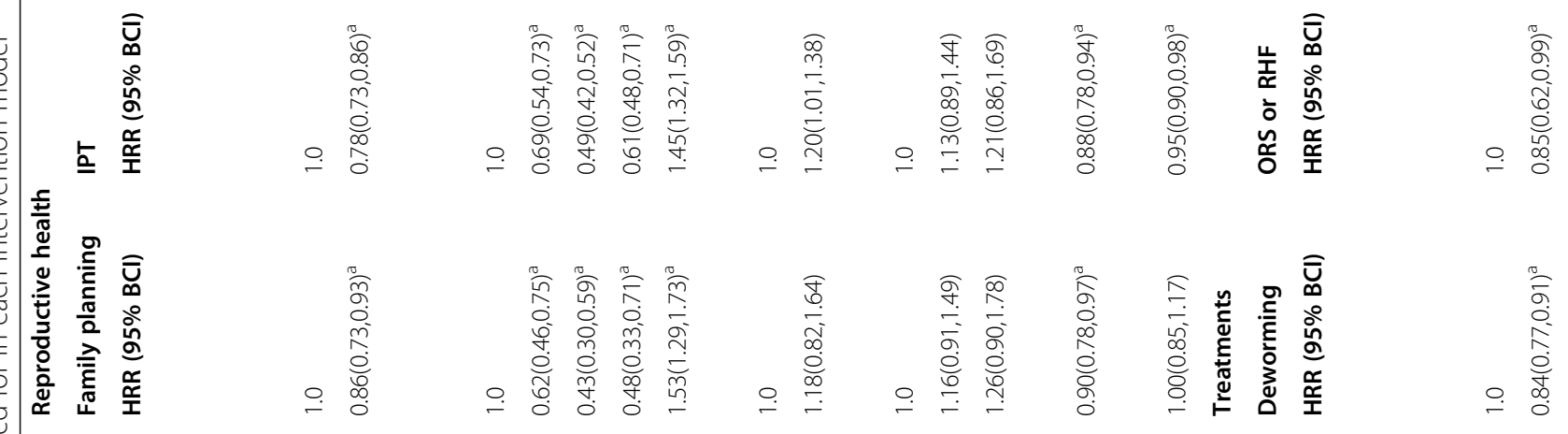

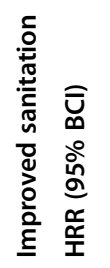
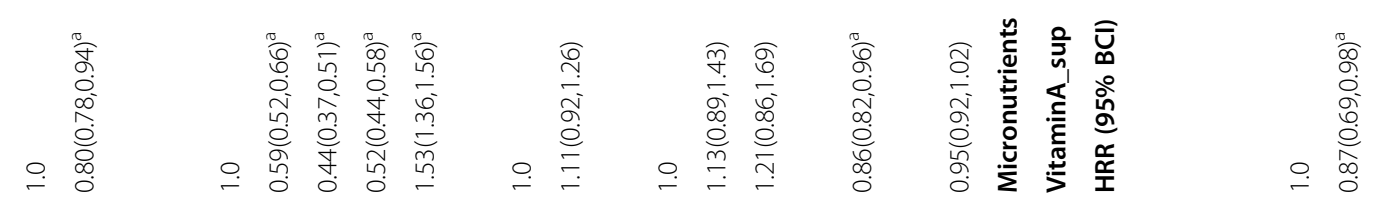

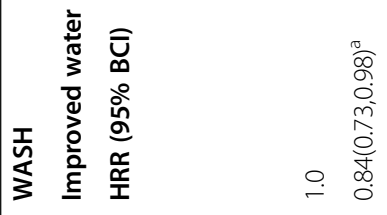
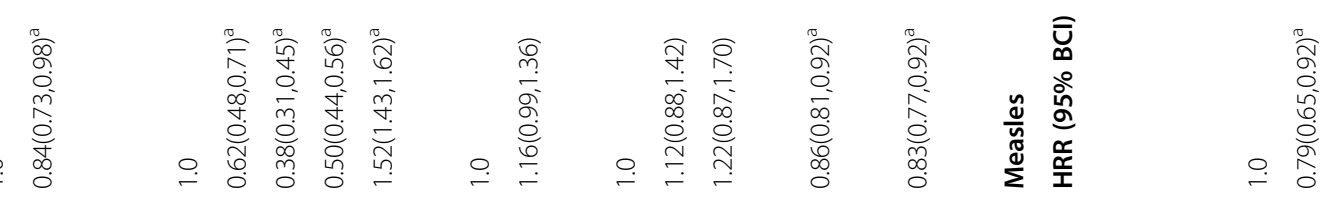

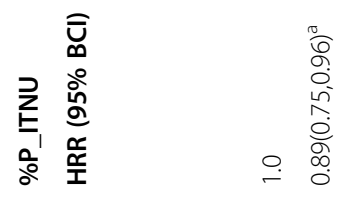

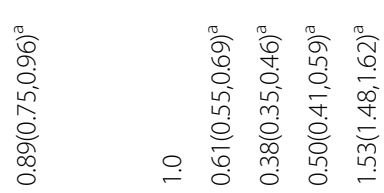

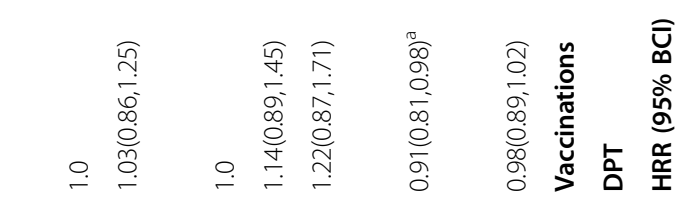

$\begin{array}{r}0 \\ 0 \\ 0 \\ 0 \\ 0 \\ 0 \\ 0 \\ 0 \\ 0 \\ 0 \\ \hline \\ \hline\end{array}$

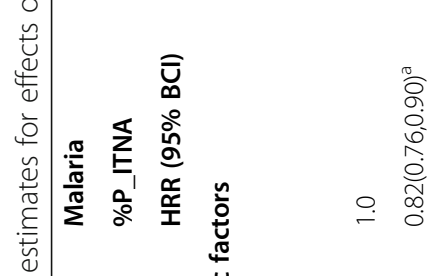

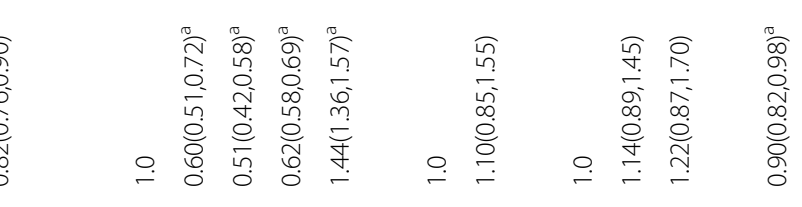

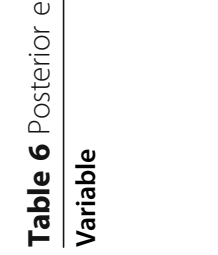

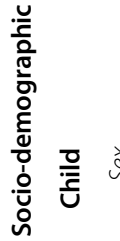

$\frac{\frac{0}{\pi}}{\frac{0}{\pi}} \stackrel{\frac{0}{\pi}}{\llcorner}$

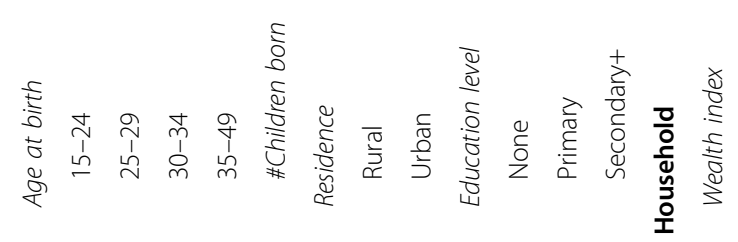
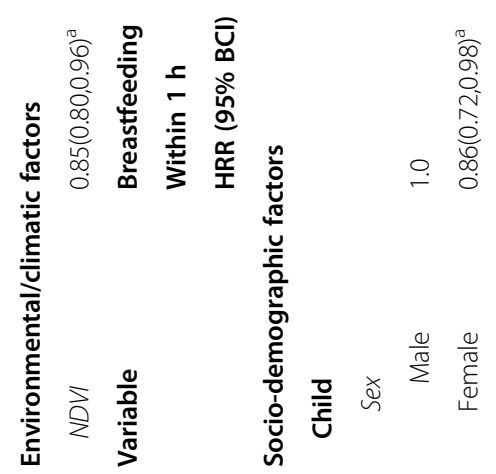


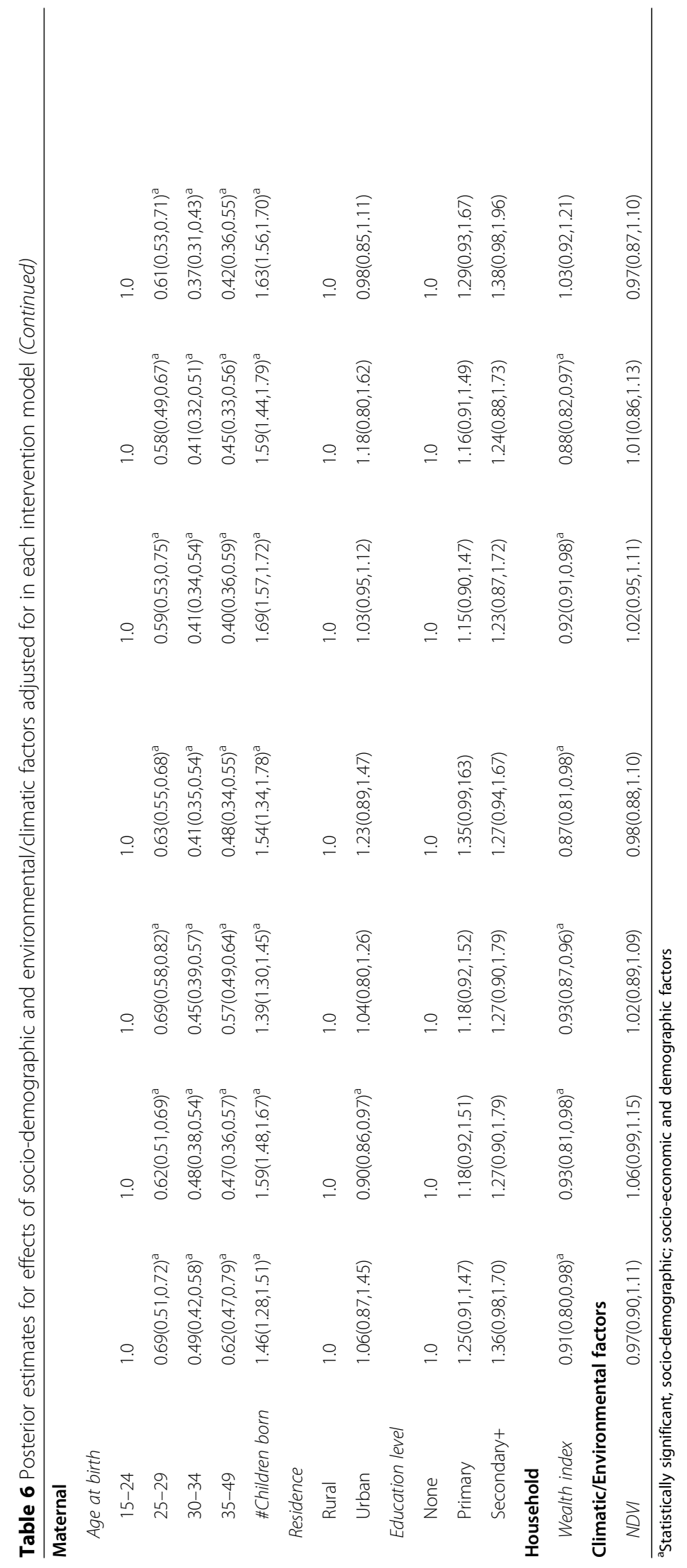


was obtained from the data. Several researchers including McGovern and Canning [27], have implemented this correction. Despite the correction, there were not significant differences between results obtained from the raw and corrected data, both in magnitude and direction. Most risk ratios were exactly the same or differed by 0.1 . This may imply that applying the correction to our data may not greatly affect the conclusions drawn based on the findings of this manuscript [27].

Another limitation of the paper is that the causal effects of health interventions on U5M cannot be identified. This is because the DHS data used are cross-sectional and can only capture cross-sectional associations rather than effects that reflect causal interpretation.

DHS surveys are designed to estimate interventions at a region level. Other combinations of strata in the DHS are the rural and urban areas. Thus, regions could be separated into urban and rural portions and estimate the coverage of interventions within each area. This would increase the number of analysis units from regions to rural and urban areas. The methodology used in this study allows the estimation of the associations of interventions at higher resolution (i.e., pixel level). However, estimates at higher resolution would require a predictive model with high resolution estimates of the coverage of interventions. Hence, there is a research need to identify predictors of intervention coverage at very high resolution.

Regardless of these limitations, our analytical approach enabled estimation of the geographical variations in the effects of health interventions on U5M, which informs countries about the effects of interventions at a subnational scale, so that appropriate interventions can be implemented and monitored over time.

\section{Conclusions}

We demonstrated that the associations of health interventions with U5M vary across regions in Uganda and identified interventions associated with largest reductions in U5M by region. These findings can guide control programmes to implement the most appropriate interventions at a local scale, especially at regions. This may reduce within country U5M inequalities and consequently result into achieving national SDG mortality targets.

The coverage of interventions associated with the highest reduction in mortality in each region should be improved. In Central 2, Mid-Western and South-West regions, ITN access should be strengthened. There is a need to increase coverage of improved sources of drinking water in Mid-North and West-Nile, while efforts should be made on improving sanitation facilities in the North-East. IPTp coverage should be scaled up in
Kampala and Mid-Eastern. ORS or RHF and postnatal care should be prioritized in Central 1 and East-Central respectively. Moreover, the Uganda government should improve the socioeconomic status of regions to enhance intervention performance and improve mortality rates.

\section{Supplementary information}

Supplementary information accompanies this paper at https://doi.org/10. 1186/s12889-019-7636-X.

Additional file 1. Bayesian geostatistical methods

\section{Abbreviations}

ACT: Artemisinin-based combination therapy; ANC: Antenatal care; ARIs: Acute respiratory infections; BCG: Bacillus Calmette Guerin; BCl: Bayesian credible interval; CA: California; DHS: Demographic and health survey; DPT: Diphtheria, pertussis and tetanus; HRR: Hazard rate ratio; IGBP: International Global Biosphere Programme; IPTp: Intermittent preventive treatment for pregnancy; IRS: Indoor residual spraying; ITN: Insecticide-treated net; LST: Land surface temperature; MIS: Malaria Indicator Survey; MODIS: Moderate resolution imaging spectroradiometer; NDVI: Normalized difference vegetation index; ORS: Recommended home fluids; RHF: Oral rehydration solution; SDG: Sustainable development goal; SRTM: Shuttle radar topographic mission; TX: Texas; U5M: Under-five mortality; UBOS: Uganda Bureau of Statistics; UK: United Kingdom; USA: United States of America; USAID: United States Agency for International Development; USGSS: United States Geological Survey-Earth Resources Observation Systems; WASH: Water, sanitation and hygiene

\section{Acknowledgements}

The authors are grateful to the DHS MEASURE for making the data available, Makerere University School of Public Health and the Swiss Tropical and Public Health Institute.

\section{Authors' contributions}

BBN conceptualized, managed and analysed data, developed and implemented the methodology, interpreted results and wrote the first draft of the manuscript. BBN also revised and incorporated reviewers' and editor's comments in the first version of the manuscript. JS reviewed and edited the manuscript. FEM and SK formulated of research goals and objectives, participated in the acquisition of project financial support, result interpretation and reviewed the first version of the manuscript. JU reviewed and edited the manuscript. PV is the lead author, conceptualized, formulated research goals and objectives, contributed to the acquisition of project financial support, lead methodology development and implementation, result interpretation and was a major contributor in writing the first draft of the manuscript, reviewing and editing. All authors read and approved the manuscript.

\section{Funding}

This research was supported and funded by the Swiss Programme for Research on Global Issues for Development project (R4D; grant number: IZ01Z0-147286) and the European Research Council advanced grant project (grant number: 323180). The funding bodies did not participate in the design of the study and data collection, analysis, and interpretation of data and in writing the manuscript.

\section{Availability of data and materials}

The datasets supporting conclusions of this article were requested from the DHS program website (www.dhsprogram.com) and can be accessed following instructions at https://dhsprogram.com/data/Access-Instructions. $\mathrm{cfm}$.

\section{Ethics approval and consent to participate}

In this research article, secondary data that were made available to us by the Uganda Bureau of Statistics and the DHS Program (www.dhsprogram.com) were used. Ethical approval was obtained by the above bodies from the Institutional Review Board of International Consulting Firm of Calverton, 
Maryland, USA, and from Makerere University School of Biomedical Sciences Higher Degrees Research and Ethics committee and the Uganda National Council for Science and Technology. Information on ethical approval is published in the 2006, 2011 DHS [2, 3] and 2009 MIS reports [12]. Consent to participate was obtained by the Uganda Bureau of Statistics and the DHS Program from the Institutional Review Board of International Consulting Firm of Calverton, Maryland, USA, and from Makerere University School of Biomedical Sciences Higher Degrees Research and Ethics committee and the Uganda National Council for Science and Technology. Interviewers read an informed consent statement to the respondents, and an interview was conducted only if respondents assented verbally. Information regarding the consent process is published in the 2006, 2011 DHS [2, 3] and 2009 MIS reports [20].

\section{Consent for publication}

Not applicable.

\section{Competing interests}

The authors declare that they have no competing interests.

\section{Author details}

'Swiss Tropical and Public Health Institute, P.O. Box, CH-4002 Basel, Switzerland. ${ }^{2}$ University of Basel, P.O. Box, CH-4003 Basel, Switzerland. ${ }^{3}$ Makerere University School of Public Health, New Mulago Hospital Complex, P.O. Box 7072, Kampala, Uganda.

Received: 7 May 2019 Accepted: 16 September 2019 Published online: 22 October 2019

\section{References}

1. Nafiu L. Determinants of under-five mortality in Abim district, Uganda. Pac J Sci Technol. 2016;17:223-8.

2. Gebretsadik S, Gabreyohannes E. Determinants of under-five mortality in high mortality regions of Ethiopia: an analysis of the 2011 Ethiopia Demographic and Health Survey data. Int J Population Res. 2016;2016:7. https://doi.org/10.1155/2016/1602761.

3. Chin B, Montana L, Basagaña X. Spatial modeling of geographic inequalities in infant and child mortality across Nepal. Health \& Place. 2011;17:929-36. https://doi.org/10.1016/j.healthplace.2011.04.006.

4. Kabagenyi R. The effect of household characteristics on child mortality in Uganda. Am J Sociol Res. 2013;3:1-5. https://doi.org/10.5923/j.sociology. 20130301.01.

5. Nasejje JB, Mwambi HG, Achia TNO. Understanding the determinants of under-five child mortality in Uganda including the estimation of unobserved household and community effects using both frequentist and Bayesian survival analysis approaches. BMC Public Health. 2015;15:1003. https://doi.org/10.1186/s12889-015-2332-y.

6. Thomson MC, Mason SJ, Phindela T, Connor SJ. Use of rainfall and sea surface temperature monitoring for malaria early warning in Botswana. Am J Tropical Med Hygiene. 2005;73:214-21. https://doi.org/10.4269/ajtmh.2005.73.214

7. Rutstein SO. Effects of preceding birth intervals on neonatal, infant and under-five years mortality and nutritional status in developing countries: evidence from the Demographic and Health Surveys. Int J Gynecol Obstet. 2005;89:S7-24. https://doi.org/10.1016/j.ijgo.2004.11.012.

8. Uganda Bureau of Statistics (UBOS) and ICF International Inc. Uganda Demographic and Health Survey 2011. Kampala, Uganda: UBOS and Calverton, Maryland: ICF International Inc.; 2012.

9. Uganda Bureau of Statistics (UBOS) and ICF Macro. Uganda Malaria Indicator Survey 2009. Calverton, Maryland, USA: UBOS and ICF Macro; 2010.

10. Lassi ZS, Mallick D, Das JK, Mal L, Salam RA, Bhutta ZA. Essential interventions for child health. Reprod Health. 2014;11:S4. https://doi.org/10. 1186/1742-4755-11-S1-S4.

11. Bbaale E. Immunization status and child survival in Uganda. African J Econ Rev. 2015;3:1-20.

12. Brenner JL, Kabakyenga J, Kyomuhangi T, Wotton KA, Pim C, Ntaro M, et al. Can volunteer community health workers decrease child morbidity and mortality in South-Western Uganda? An impact evaluation. PLoS One. 2011; 6:e27997. https://doi.org/10.1371/journal.pone.0027997.

13. Nankabirwa V, Tumwine JK, Mugaba PM, Tylleskär T, Sommerfelt H. Child survival and BCG vaccination: a community based prospective cohort study in Uganda. BMC Public Health. 2015;15:175. https://doi.org/10.1186/s12889-015-1497-8.
14. Masanja H, de Savigny D, Smithson P, Schellenberg J, John T, Mbuya C, et al. Child survival gains in Tanzania: analysis of data from Demographic and Health Surveys. Lancet. 2008;371:1276-83. https://doi.org/10.1016/S01406736(08)60562-0.

15. Vaugelade J, Pinchinat S, Guiella G, Elguero E, Simondon F. Non-specific effects of vaccination on child survival: prospective cohort study in Burkina Faso. BMJ. 2004;329:1309. https://doi.org/10.1136/bmj.38261.496366.82.

16. Aaby P, Martins CL, Garly M-L, Bale C, Andersen A, Rodrigues A, et al. Nonspecific effects of standard measles vaccine at 4.5 and 9 months of age on childhood mortality: randomised controlled trial. BMJ. 2010;341:c6495. https://doi.org/10.1136/bmi.c6495.

17. Higgins JPT, Soares-Weiser K, López-López JA, Kakourou A, Chaplin K, Christensen $\mathrm{H}$, et al. Association of BCG, DTP, and measles containing vaccines with childhood mortality: systematic review. BMJ. 2016;355:15170. https://doi.org/10.1136/bmj.i5170.

18. Ent M, Brown DW, Hoekstra EJ, Christie A, Cochi SL. Measles mortality reduction contributes substantially to reduction of all cause mortality among children less than five years of age, 1990-2008. J Infect Dis. 2011; 204:S18-23. https://doi.org/10.1093/infdis/jir081.

19. WHO. Recommended routine immunizations for children. Geneva: World Health Organization; 2018

20. Babirye JN, Engebretsen IMS, Makumbi F, Fadnes LT, Wamani H, Tylleskar T, et al. Timeliness of childhood vaccinations in Kampala Uganda: a community-based cross-sectional study. PLoS One. 2012;7:e35432. https:// doi.org/10.1371/journal.pone.0035432.

21. Akmatov MK, Kretzschmar M, Krämer A, Mikolajczyk RT. Timeliness of vaccination and its effects on fraction of vaccinated population. Vaccine. 2008;26:3805-11. https://doi.org/10.1016/j.vaccine.2008.05.031.

22. Fadnes LT, Nankabirwa V, Sommerfelt H, Tylleskär T, Tumwine JK, Engebretsen IMS. Is vaccination coverage a good indicator of ageappropriate vaccination? a prospective study from Uganda. Vaccine. 2011; 29:3564-70. https://doi.org/10.1016/j.vaccine.2011.02.093.

23. Ezeh OK, Agho KE, Dibley MJ, Hall J, Page AN. The impact of water and sanitation on childhood mortality in Nigeria: evidence from Demographic and Health Surveys, 2003-2013. Int J Environ Res Public Health. 2014;11: 9256-72. https://doi.org/10.3390/ijerph110909256.

24. Kaberuka W, Mugarura A, Tindyebwa J, Bishop DS. Factors determining child mortality in Uganda. Int J Soc Econ. 2017:44:633-42. https://doi.org/10.1108/ IJSE-08-2015-0201.

25. Kanmiki EW, Bawah AA, Agorinya I, Achana FS, Awoonor-williams JK, Oduro $A R$, et al. Socio-economic and demographic determinants of under-five mortality in rural northern Ghana. BMC Int Health Hum Rights. 2014;14:24. https://doi.org/10.1186/1472-698X-14-24.

26. Ezeh OK, Agho KE, Dibley MJ, Hall JJ, Page AN. Risk factors for postneonatal, infant, child and under-5 mortality in Nigeria: a pooled cross-sectional analysis. BMJ Open. 2015;5:e006779. https://doi.org/10.1136/bmjopen-2014-006779.

27. McGovern ME, Canning D. Vaccination and all-cause child mortality from 1985 to 2011: global evidence from the demographic and health surveys. Am J Epidemiol. 2015;182:791-8. https://doi.org/10.1093/aje/kwv125.

28. Reniers G, Eaton J. Refusal bias in HIV prevalence estimates from nationally representative seroprevalence surveys. AIDS. 2009;23:621-9. https://doi.org/ 10.1097/QAD.0b013e3283269e13.

\section{Publisher's Note}

Springer Nature remains neutral with regard to jurisdictional claims in published maps and institutional affiliations.

Ready to submit your research? Choose BMC and benefit from:

- fast, convenient online submission

- thorough peer review by experienced researchers in your field

- rapid publication on acceptance

- support for research data, including large and complex data types

- gold Open Access which fosters wider collaboration and increased citations

- maximum visibility for your research: over $100 \mathrm{M}$ website views per year

At BMC, research is always in progress.

Learn more biomedcentral.com/submission 